\title{
Disposición hacia el pensamiento crítico, nivel académico, género y resolución de problemas en educación secundaria
}

\author{
Disposition Towards Critical Thinking, Academic Level, Gender and \\ Problem Solving in Secondary Education
}
Disposição ao pensamento crítico, nível acadêmico, gênero e resolução de problemas no ensino médio

\author{
José Piquer Ramada * id Joan Josep Solaz-Portolés* * id Vicente Sanjosé López * * *
}

\section{OPEN ACCESS $\boldsymbol{\Omega}$ \\ DOI: http://dx.doi.org/10.18634/sophiaj. 17v.1i.1040}

Información del artículo

Recibido: octubre 06 de 2020

Revisado: enero 12 de 2021

Aceptado: enero 28 de 2021

Publicado: abril 15 de 2021

Palabras clave: pensamiento crítico, nivel de enseñanza, estudiantes de secundaria, resolución de problemas, educación.

Keywords: thinking critical, educational level, secondary school students, problem solving, education.

Palavras-chave: pensamento crítico, nível educacional, alunos do ensino médio, resolução de problemas, educação

Cómo citar: / how cite:

Piquer, J., Solaz-Portoléz, J.J., Sanjosé V. (2021) Disposición hacia el pensamiento crítico, nivel académico, género y resolución de problemas en educación secundaria. Sophia 17 (1), e1040

Sophia-Educación, volumen 17 número 1. enero/junio 2021. Versión español
* Departamento de Didáctica de las Ciencias Experimentales y Sociales Universitat de València, València, España. http://orcid.org/0000-0002-5405-9521pirajo@alumni.uv.es **Doctor en Ciencias Químicas (Programa Investigación en Didáctica de las Ciencias). Profesor titular de Didáctica de las Ciencias Experimentales. Departamento de Didáctica de las Ciencias Experimentales y Sociales de la Universitat de València. Valencia, España. Joan.Solaz@uv.es Orcid id: https:// orcid.org/0000-0003-4690-6556***Doctor en Ciencias Físicas. Catedrático de Didáctica de las Ciencias Experimentales. Departamento de Didáctica de las Ciencias Experimentales y Sociales de la Universitat de València. Valencia, España. Vicente.Sanjose@uv.es Orcid id: https://orcid. org/0000-0003-3806-1717

RESUMEN

El propósito de este estudio fue investigar la posible influencia de la disposición hacia el pensamiento crítico, el nivel académico y el género (variables independientes) sobre el desempeño en resolución de problemas de estudiantes de secundaria (variable dependiente). Han participado 114 estudiantes de educación secundaria españoles, 55 chicos y 59 chicas, de $3^{\circ}$ y $4^{\circ}$ de ESO y de $1^{\circ}$ de Bachillerato $\left(9^{\circ}, 10^{\circ}\right.$ y $11^{\circ}$ grado, respectivamente). A todos ellos se les ha administrado un cuestionario sobre disposición hacia el pensamiento crítico y una prueba de resolución de problemas (un problema de las pruebas PISA). De la matriz de correlaciones producto-momento de Pearson entre variables y del análisis de regresión backward stepwise efectuado se han derivado las siguientes conclusiones: 1) la disposición hacia el pensamiento crítico (esto es, el potencial uso del pensamiento crítico) y el género parecen tener un insignificante peso en el desempeño en la resolución de problemas; y 2) el nivel académico muestra una contribución muy destacada en el rendimiento en la resolución de problemas. 
Copyright 2021 Universidad La Gran Colombia

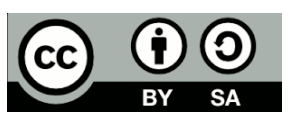

Conflicto de interés:

Los autores declaran no tener ningún conflicto de interés.

Correspondencia de autor:

pirajo@alumni.uv.es

Joan.Solaz@uv.es

Vicente.Sanjose@uv.es

\section{ABSTRACT}

The purpose of this study was to investigate the possible influence of critical thinking disposition, academic level and gender (independent variables) on the problem-solving performance of high school students (dependent variable). A total of 114 Spanish secondary school students participated, 55 boys and 59 girls, from the 3rd and 4th years of Compulsory Secondary Education (ESO) and the 1st year of High School (9th, 10th and 11th grade, respectively). All of them were administered a questionnaire on critical thinking disposition and a problem-solving test (a PISA test problem). From the Pearson product-moment correlation matrix between variables and the backward stepwise regression analysis performed, the following conclusions have been derived: 1 ) the disposition towards critical thinking (i.e., the potential use of critical thinking) and gender seem to have an insignificant weight on problem-solving performance; and 2) academic level shows a very prominent contribution to problem-solving performance.

\section{RESUMO}

O objetivo deste estudo foi investigar a possível influência da disposição para o pensamento crítico, nível acadêmico e gênero (variáveis independentes) no desempenho na resolução de problemas de alunos do ensino médio (variável dependente). Participaram 114 alunos espanhóis do ensino secundário, 55 garotos e 59 garotas, do $3^{\circ}$ e $4^{\circ}$ do ESO e do $1^{\circ}$ de ensino médio $\left(9^{\circ}, 10^{\circ}\right.$ e $11^{\circ}$ ano, respectivamente). Todos eles receberam um questionário sobre a disposição para o pensamento crítico e um teste de resolução de problemas (um problema nos testes PISA). A partir da matriz de correlação produtomomento de Pearson entre as variáveis e a análise de regressão backward stepwise realizada, as seguintes conclusões foram derivadas: 1) a disposição para o pensamento crítico (isto é, o uso potencial do pensamento crítico) e o gênero parecem têm peso insignificante no desempenho de resolução de problemas; e 2) o nível acadêmico mostra uma contribuição muito importante para o desempenho na resolução de problemas. 


\section{Introducción}

De acuerdo con Fisher (2011) el pensamiento crítico puede definirse como el conjunto de procesos que permiten tomar una decisión bien fundamentada en cuestiones o problemas conflictivos, aunque parece que resulta difícil establecer su naturaleza o si sus características tienen un carácter general (Phillips \& Bond, 2004). Todo apunta a que el pensamiento crítico está relacionado con las habilidades que están presentes en la resolución de problemas, toma de decisiones, inferencias, pensamiento divergente, pensamiento evaluativo, procesos de razonamiento o transferencia de conocimientos (Carvalho et al., 2015). Kuhn (1999) fue una de las primeras en proponer un modelo de desarrollo de pensamiento crítico. En dicho modelo se defiende que el pensamiento crítico se asienta sobre tres pilares cognitivos, uno metacognitivo, uno metaestratégico y uno epistemológico. Investigaciones más recientes sitúan al pensamiento crítico dentro de uno de los componentes de autorregulación del aprendizaje, en concreto dentro de las estrategias cognitivas (Phan, 2010; Schraw, Crippen, \& Hartley, 2006).

Pensar críticamente es una competencia básica que todos los ciudadanos deberían desarrollar en la educación obligatoria para poder participar activamente en la sociedad (Ten Dam \& Volman, 2004). Así, por ejemplo, la creatividad está muy vinculada a las destrezas de pensamiento crítico (Tsai, 2019). Sin embargo, no todos los estudiantes pueden llegar a desarrollar convenientemente esta competencia, ni todos los profesores emplean metodologías que posibiliten desarrollar el pensamiento crítico en sus estudiantes (Pithers \& Soden, 2000). Ku y Ho (2010) examinaron el papel de las destrezas metacognitivas en el desarrollo del pensamiento crítico, y encontraron que la implicación de los estudiantes en actividades que supongan la aplicación de estrategias de evaluación y planificación, conducen a una mejora del pensamiento crítico. Magno (2010) analizó el efecto de las destrezas metacognitivas sobre el pensamiento crítico mediante un modelo de ecuaciones estructurales, y concluyó que dichas destrezas intervienen de forma significativa en el pensamiento crítico. Por otra parte, se ha comprobado que las destrezas metacognitivas progresan a medida que se avanza en la educación secundaria (Van der Stel, Veenman, Deelen, \& Haenen, 2010; Veenman, \& Spaans, 2005). En consecuencia, cabe esperar que el pensamiento crítico mejore a lo largo de educación secundaria.

Ennis (1987) ya señalaba en los años 80 que las estrategias de pensamiento crítico incluían habilidades necesarias en la resolución de problemas. Posteriormente, Facione (1990) llevó a cabo un estudio para identificar el pensamiento crítico en estudiantes universitarios y destacó que éste está ligado actividades de alto nivel cognitivo, a la toma de decisiones y a la resolución de problemas. Pushkin (2007) ofreció una explicación de cómo el pensamiento crítico ayuda en la resolución de problemas basada en dos tipos de conocimiento: el conocimiento de estrategias y el conocimiento situacional. Friede, Irani, Rhoades, Fuhrman y Gallo (2008) determinaron destrezas de pensamiento crítico (a través de un análisis de contenido de un protocolo think-aloud) y comprobaron que éstas contribuyen poco, pero de forma significativa, a la resolución de problemas. Estos autores afirman que son necesarias más investigaciones que clarifiquen cómo y dónde afecta el pensamiento crítico en la resolución de problemas. En el trabajo de Özyurt (2015) se examinaron las investigaciones realizadas sobre la incidencia del pensamiento crítico en la resolución de problemas que, según indica el autor, son muy pocas y centradas en dos grupos: profesorado (en activo o en formación) y los estudiantes de enfermería.

Como apunta Butler (2012), el pensamiento crítico es un constructo complejo y, como tal, difícil de evaluar. Con frecuencia se distingue entre destrezas de pensamiento crítico y disposición hacia el pensamiento crítico. En el estudio de Facione (1990) se vio que el pensamiento crítico está compuesto de las destrezas de pensamiento crítico y de la disposición hacia el pensamiento crítico y, más tarde, Norris (1994) concluyó que la disposición hacie el pensamiento crítico era necesaria para un uso apropiado de las destrezas de pensamiento crítico. Sin embargo, Halpern (2003) considera que tanto las destrezas de pensamiento crítico como la disposición hacia su uso se involucran en un mismo proceso de razonamiento. De hecho, Rikectts y Rudd (2005) encontraron una correlación positiva y significativa entre ambas variables.

Los resultados del estudio de Tümkaya, Aybek y Aldaş (2009) muestran que tener mejor disposición hacia el pensamiento crítico está asociado a mejores habilidades en la resolución de problemas. Özyurt (2015) también encontró una correlación significativa entre ambas variables, pero de bajo nivel. En ambos casos los participantes fueron estudiantes univesitarios. Por su parte Kawashima y Shiomi concluyeron que el género no produce diferencias significativas en la disposición de estudiantes de secundaria hacia el pensamiento crítico (Kawashima \& Shiomi, 2007). 
Mejorar el rendimiento en la resolución de problemas continúa siendo un objetivo central de los profesores de ciencias y matemáticas, así como de los investigadores en didáctica de las ciencias y las matemáticas. Para lograr este objetivo, Lee, Tang, Goh y Chia (2001) propusieron que se tenían que desarrollar habilidades propias de la resolución de problemas en actividades específicas en el aula, y que era necesario conocer y abordar las dificultades de los estudiantes durante la resolución. Todo esto no puede conseguirse si se desconocen los procesos cognitivos y los conocimientos que se ponen en juego durante la resolución (Solaz-Portolés \& Sanjosé, 2008). Se ha comprobado en diversos trabajos que el rendimiento en resolución de problemas mejora a medida que avanza en la educación secundaria, de forma acorde con la mejora de conocimientos y estrategias que se genera (Alabau, Solaz-Portolés, \& Sanjosé, 2020; García-Gallego, Sanjosé, y Solaz-Portolés, 2015; Mugarra-Soldevila, Solaz-Portolés, Caurín-Alonso, 2014). Por otra parte, los metaanálisis efectuados por Hyde, Lindberg, Linn, Ellis y Williams (2008) y Lindberg, Hyde, Petersen y Linn (2010), ratifican que chicas y chicos tienen un desempeño muy parecido en pruebas de resolución de problemas. Sin embargo, en la revisión bibliográfica realizada por Zhu (2007) aparecen investigaciones en las que el género produce diferencias significativas en la resolución de problemas, concretamente los hombres parece que superan a las mujeres. De hecho, Gallagher et al. (2000), comprobaron que los chicos superan a las chicas en resolución de problemas complejos.

Como se ha visto, el pensamiento crítico interviene activamente en los procesos de resolución de problemas. Además, los procesos de resolución pueden verse afectados tanto por el género como por el nivel académico de los estudiantes que los llevan a cabo. Por otro lado, se ha indicado la dificultad de evaluar el pensamiento crítico, cosa que no ocurre con la disposición hacia el pensamiento crítico, íntimamente asociada a las destrezas de pensamiento crítico. Dada la escasez de trabajos en la educación secundaria donde se analice la relación entre desempeño en resolución de problemas y la disposición hacia el pensamiento crítico, el problema que se quiere investigar en este estudio es la posible influencia de la disposición hacia el pensamiento crítico, el nivel de formación académica y el género (variables independientes) sobre el desempeño en resolución de problemas de los estudiantes de secundaria (variable dependiente). En concreto, los objetivos del estudio fueron: a) Evaluar la relación entre cada una de las variables independientes y la variable dependiente; b) Examinar la contribución relativa de las tres variables independientes a la variabilidad de la variable dependiente; y c) Determinar la variable independiente que mejor predice el desempeño en resolución de problemas.

\section{Metodología}

\section{Participantes}

Han participado 31 alumnos de $3^{\circ}$ de Educación Secundaria Obligatoria -ESO- (9º grado, 14-15 años), 37 de $4^{\circ}$ de ESO ( $10^{\circ}$ grado, $15-16$ años), y 46 de $1^{\circ}$ de Bachillerato ( $11^{\circ}$ grado, 16-17 años). Del total de 114 alumnos, 55 eran chicos y 59 chicas. Se trata de una muestra de conveniencia de estudiantes que provienen de un instituto público de educación secundaria de Valencia (España). Estos sujetos no presentan, a priori, características especiales que los diferencien de otros grupos de los respectivos cursos. No obstante, los resultados no pueden todavía ser extrapolados a toda la población estudiantil de esos cursos con fiabilidad.

\section{Instrumentos}

Se ha utilizado el cuestionario Critical Thinking Motivation Scales (Valenzuela, Nieto, \& Saiz, 2011) para medir la disposición hacia el pensamiento crítico. Es un instrumento para medir el grado de motivación que pueden tener las personas para pensar críticamente. Tiene un total de 19 ítems que se agrupan en 5 bloques o factores, todos ellos referidos al pensamiento crítico: expectativas, alcance, utilidad, coste e interés. Así, algunas proposiciones recogidas en los ítems están referidas a lo que se espera del pensamiento crítico (expectativas), otras al valor que se le otorga a este (valor). También hay proposiciones sobre la importancia del uso de esta forma de pensar (utilidad), sobre los perjuicios que se está dispuesto a aceptar (coste) y del provecho que se espera de él (interés). Se tradujo al castellano y se revisó y adaptó (tras darlo a leer a tres estudiantes de secundaria) por dos profesores universitarios y un profesor de secundaria. A continuación, se ofrece un ejemplo de cada factor:

- Expectativas: «Puedo aprender cómo razonar correctamente mejor que la mayoría de mis compañeros"

- Valor: «Para mí, es importante usar mis habilidades intelectuales correctamente» 
- Utilidad: «El pensamiento crítico es útil en el día a día»

- Interés: "Me gusta razonar de una forma rigurosa"

- Coste: "Yo estoy dispuesto a sacrificar gran parte de mi tiempo y esfuerzo con el fin de mejorar mi forma de razonar"

El cuestionario utiliza una escala tipo Likert con seis niveles de respuesta, que van desde completamente en desacuerdo (valor 1), hasta completamente de acuerdo (valor 6). El orden de los ítems en el cuestionario fue aleatorizado.

También se administró una prueba de resolución de problemas que recoge un problema de las pruebas PISA del año 2003 (Inecse, 2005), con dos apartados. En el Anexo 1 puede verse dicho problema. Con esta prueba se pretendió medir el rendimiento de resolución de problemas de cada estudiante. Para su evaluación se empleó la rúbrica de evaluación que viene adjunta a los problemas en Inecse (2005). El primer apartado tiene una puntuación máxima de 1 punto, y el segundo de 3 puntos. Por tanto, la calificación de la prueba de resolución de problemas oscila entre 0 y 4 puntos.

Se administró el cuestionario sobre disposición hacia el pensamiento crítico durante los últimos 25 minutos de una sesión de clase normal. Se pidió a los alumnos que leyeran con atención todos los ítems del cuestionario y que respondieran con la máxima sinceridad. La prueba de resolución de problemas se administró en otra sesión de clase normal y ocupó entre 15 y 20 minutos minutos.

La calificación de la prueba de resolución de problemas se hizo por dos autores del presente trabajo mediante la aplicación de la rúbrica de las pruebas PISA (Coeficiente Kappa de Cohen 0.93). Tanto la calificación de esta prueba como la obtenida en el cuestionario se trasladó a una hoja de cálculo Excel y, a continuación, se llevaron a cabo los análisis estadísticos.

\section{Resultados}

Primeramente, se ha calculado el coeficiente alfa de Cronbach del cuestionario sobre disposición hacia el pensamiento crítico, que ha proporcionado un valor de 0.82 , que nos indica un nivel de fiabilidad alto. Las medias aritméticas y la desviación estándar de las puntuaciones obtenidas en la prueba de resolución de problemas y en el cuestionario (suma de las puntuaciones en todos los ítems del mismo) en función del nivel académico y del género, se muestran en la tabla 1. La media global de disposición hacia el pensamiento crítico es de 86.17 puntos (puntuación máxima en el cuestionario 114 puntos), una puntuación media-alta. En la tabla 1 puede verse que las medias en esta variable cubren un rango desde los 83.33 a 89.21 puntos. Por su parte, la media global de la prueba de resolución de problemas se sitúa en 2.10 puntos (puntuación máxima en la prueba 4 puntos), una puntuación media. Las medias en esta variable fluctúan entre 0.81 y 3.35 puntos.

Tabla 1. Estadística descriptiva de las variables en el estudio

\begin{tabular}{|c|c|c|c|c|}
\hline Variable & $\begin{array}{c}\text { Nivel } \\
\text { académico }\end{array}$ & Género & Media & S.D. \\
\hline \multirow{6}{*}{$\begin{array}{l}\text { Disposición } \\
\text { Pensamiento } \\
\text { Crítico (Punt. } \\
\text { máxima 114) }\end{array}$} & $3^{\circ} \mathrm{ESO}$ & Chico & 83.33 & 13.39 \\
\hline & & Chica & 88.31 & 12.99 \\
\hline & $4^{\circ} \mathrm{ESO}$ & Chico & 84.09 & 9.52 \\
\hline & & Chica & 86.64 & 11.78 \\
\hline & $1^{\circ} \mathrm{BAC}$ & Chico & 83.88 & 17.04 \\
\hline & & Chica & 89.21 & 10.24 \\
\hline \multirow{6}{*}{$\begin{array}{l}\text { Resolución de } \\
\text { problemas (Punt. } \\
\text { máxima 4) }\end{array}$} & $3^{\circ} \mathrm{ESO}$ & Chico & 1.27 & 1.10 \\
\hline & & Chica & 0.81 & 1.05 \\
\hline & $4^{\circ} \mathrm{ESO}$ & Chico & 1.70 & 1.15 \\
\hline & & Chica & 2.07 & 0.92 \\
\hline & $1^{\circ} \mathrm{BAC}$ & Chico & 3.35 & 0.86 \\
\hline & & Chica & 2.83 & 1.00 \\
\hline
\end{tabular}


La tabla 2 presenta la matriz de correlaciones producto-momento de Pearson de las variables implicadas en el estudio, incluyendo el nivel académico (se ha tomado valor 0 para $3^{\circ}$ de ESO, valor 1 para $4^{\circ}$ de ESO, y valor 2 para $1^{\circ}$ de Bachillerato) y el género (considerando valor 0 para los chicos y valor 1 para las chicas). Se puede observar que la resolución de problemas y la disposición hacia el pensamiento crítico presentan una correlación positiva, pero pequeña y no significativa $(r=0.15, p>.05)$, al igual que la disposición hacia el pensamiento crítico y el género $(r=0.18, p>$.05). La correlación entre resolución de problemas y el nivel académico es muy alta $(r=0.62, p<.00001)$. No hay correlación entre resolución de problemas y género $(r=0.00)$, ni entre disposición hacia el pensamiento crítico y nivel académico $(r=0.05)$.

Tabla 2. Correlaciones producto-momento de Pearson entre variables

\begin{tabular}{lcccc}
\hline & $\begin{array}{c}\text { Res. } \\
\text { Problemas }\end{array}$ & $\begin{array}{c}\text { Disposición } \\
\text { P.C. }\end{array}$ & $\begin{array}{c}\text { Nivel } \\
\text { académico }\end{array}$ & Género \\
\hline Res. Problemas & 1 & 0.15 & $0.62^{*}$ & 0.00 \\
Disposición P.C. & 1 & 0.05 & 0.18 \\
Nivel académico & & 1 & 0.11 \\
\hline
\end{tabular}

$* p<0.00001 \quad$ Fuente: elaboración propia

Con la finalidad de estudiar si la puntuación obtenida en la prueba de resolución de problemas puede ser predicha a partir de las otras variables puestas en juego en esta investigación, se efectuó un análisis de regresión múltiple. En este análisis se tomó como variable dependiente, o criterio, la puntuación en la prueba de resolución de problemas, y como variables independientes, o predictores, la puntuación de disposición hacia el pensamiento crítico, el nivel académico y el género. El análisis de regresión se realizó paso a paso con el método hacia atrás (backward stepwise). La tabla 3 resume el contraste de regresión (análisis de varianza) para verificar si las variables independientes están relacionadas linealmente (influyen de forma conjunta y lineal) con la variable dependiente, esto es, aportan información en la explicación de la variable dependiente. El nivel de significación de $\mathrm{F}$ es inferior a 0.00001, consiguientemente el modelo de regresión es estadísticamente significativo para explicar la variable dependiente o criterio. En cuanto a la bondad del ajuste, el cuadrado del coeficiente de correlación múltiple $\left(\mathrm{R}^{2}\right)$ es 0.394 , que indica que las tres variables independientes o predictores dan cuenta del 39.4\% de la varianza de la puntuación de la variable criterio (creencias sobre ciencia). El 60.6\% restante debe ser explicado por otras variables no contempladas en este experimento y por la varianza del error.

Tabla 3. Análisis de varianza del modelo de regresión múltiple

\begin{tabular}{lccccccc}
\hline Fuente & $\begin{array}{c}\text { Grados de } \\
\text { libertad }\end{array}$ & $\begin{array}{c}\text { Suma de } \\
\text { cuadrados }\end{array}$ & $\begin{array}{c}\text { Medias } \\
\text { cuadráticas }\end{array}$ & Valor de $\mathbf{F}$ & $\mathbf{p}$ & $\mathbf{R}^{\mathbf{2}}$ \\
\hline Modelo & 1 & 76.08 & 76.08 & 71.10 & $<0.00001$ & 0.394 \\
Error & 112 & 119.86 & 1.07 & & & \\
Total & 113 & 195.94 & 1.73 & & & \\
\hline
\end{tabular}

Fuente: elaboración propia 
La tabla 4 muestra los coeficientes de regresión de las tres variables independientes en la ecuación de regresión. Obsérvese que hay dos predictores cuyo nivel de significación no es estadísticamente significativo. También se ofrecen los factores de inflación de la varianza, que permiten estimar el nivel de multicolinealidad entre los predictores del modelo de regresión. Todos son muy poco mayores de 1, lo que indica un grado de colinealidad muy bajo.

Tabla 4. Coeficientes del análisis de regresión para la predicción del desempeño en la resolución de problemas

\begin{tabular}{lccccc}
\hline & $\begin{array}{c}\text { Coef. de } \\
\text { regresión no } \\
\text { estandarizado }\end{array}$ & Error típico & Prueba t & $\mathbf{p}$ & $\begin{array}{c}\text { Factol } \\
\text { inflaciór } \\
\text { varianz }\end{array}$ \\
\hline Intersección & -0.15 & 0.69 & -0.22 & 0.827 & \\
$\begin{array}{l}\text { Disposición } \\
\text { P. Crítico }\end{array}$ & 0.01 & 0.01 & 1.78 & 0.078 & 1.0 \\
$\begin{array}{l}\text { Nivel } \\
\text { académico }\end{array}$ & 1.01 & 0.12 & 8.50 & $<0.00001$ & 1.0 \\
Género & -0.24 & 0.20 & -1.22 & 0.225 & $1.0 !$ \\
\hline
\end{tabular}

Fuente: elaboración propia

Como se ha dicho el análisis regresión efectuado fue paso a paso hacia atrás (backward stepwise). En este tipo de regresión, las variables predictoras se van eliminando en cada paso cuando su significación supera cierto límite (en este caso, $p>$.05). En el primer paso se eliminó la variable género y en el segundo la variable disposición hacia el pensamiento crítico. En ambos casos no se observó cambio apreciable en la $\mathrm{R}^{2}$ (en la primera eliminación pasa de 0.394 a 0.391, y en la segunda a 0.388), es decir, se corrobora que estas variables no contribuyen de forma significativa a la explicación de la varianza de la puntuación de la prueba de la resolución de problemas. En la tabla 5 aparecen los resultados en el último paso del análisis.

Tabla 5. Resultados del análisis de regresión backward stepwise para la predicción del nivel de creencias sobre ciencia en el último paso

\begin{tabular}{lccccc}
\hline & $\begin{array}{c}\text { Coef. de regresión } \\
\text { no estandarizado }\end{array}$ & Error típico & Prueba t & $\mathbf{p}$ \\
\hline Intersección & 0.96 & 0.17 & 5.76 & $<.00001$ \\
Nivel académico & 1.01 & 0.12 & 8.43 & $<.00001$ \\
\hline
\end{tabular}

Por tanto, la ecuación de regresión que relaciona la puntuación de la prueba de resolución de problemas (PRP), o desempeño en resolución de problemas, y el único predictor significativo, el nivel académico (NA), es PRP = $0.96+1.01$ NA. 


\section{Discusión de resultados}

Se han de resaltar las elevadas puntuaciones obtenidas en el cuestionario de disposición hacia el pensamiento crítico que, como se puede observar en la tabla 1, se sitúan en una franja media-alta de puntuación. Contrastan estos resultados con los obtenidos por Zoller et al. (2000) con un instrumento muy diferente (largo, de 75 ítems), donde el nivel que daban estudiantes italianos e israelíes del último curso de secundaria (17-18 años) era medio (alrededor de 56 puntos, en una escala de puntuación máxima 100). Parece que los estudiantes que han participado creen tener una gran disposición hacia el pensamiento crítico. Las puntuaciones en la prueba de resolución de problemas, que van desde un nivel bajo en $3^{\circ}$ de ESO $\left(9^{\circ}\right.$ grado) hasta un nivel alto en $1^{\circ}$ de Bachillerato $\left(11^{\circ}\right.$ grado), son semejantes a las de otro trabajo donde se empleó el mismo problema (Pellicer, Solaz-Portolés, \& Sanjosé, 2019).

En cuanto a la variable género, los valores de los coeficientes de correlación de Pearson y el análisis de regresión lineal realizado indican que su influencia en el rendimiento de resolución de problemas es insignificante. Es decir, que en la educación secundaria chicos y chicas no presentan diferencias significativas en el desempeño en la resolución de problemas, como también ocurrió en el estudio de Doktor y Heller (2008). Estos resultados también son congruentes con los obtenidos por Alabau, Solaz-Portolés y Sanjosé (2020) y Hyde y Mertz (2009). Estas dos últimas autoras llegan a afirmar que las chicas han alcanzado la paridad con los chicos en la resolución de problemas. Sin embargo, Hyde et al. (2008) subrayan que los chicos hacen significativamente mejor los problemas complicados en los comienzos de la educación secundaria y que esta circunstancia podría explicar la escasa presencia de mujeres en ciertas titulaciones del área STEM.

La tabla de correlaciones producto-momento de Pearson (tabla 2) nos muestra que el nivel académico tiene un coeficiente de correlación muy alto con el rendimiento en resolución de problemas, y muy bajo con la disposición hacia el pensamiento crítico. En análisis de regresión, su contribución relativa a la predicción del desempeño en la resolución de problemas es la más importante de todas las variables y, de hecho, la única significativa. Este resultado es coherente con los resultados de diferentes investigaciones donde el nivel académico aparece asociado a la mejora en la resolución de problemas de los estudiantes ((Alabau, SolazPortolés, \& Sanjosé, 2020; García-Gallego, Sanjosé, y Solaz-Portolés, 2015; Mugarra-Soldevila, Solaz-Portolés, Caurín-Alonso, 2014). Por otro lado, la casi nula correlación entre nivel académico y disposición hacia el pensamiento crítico, o, dicho de otro modo, que el aumento del nivel académico no altera la disposición hacia el pensamiento crítico, concuerda con los resultados obtenidos por Özyurt (2015) con estudiantes universitarios. No obstante, entra en contradicción con los estudios que ratifican la mejora que se produce en las destrezas metacognitivas a medida que se avanza en la educación secundaria (Van der Stel, Veenman, Deelen, \& Haenen, 2010; Veenman \& Spaans, 2005).

Finalmente, el coeficiente de correlación entre la disposición hacia el pensamiento crítico y el desempeño en la resolución de problemas ha resultado ser positivo, bajo y no significativo. En el análisis de regresión su contribución relativa a la predicción del desempeño en la resolución de problemas no ha sido significativa, y su aportación a la explicación de la varianza de la variable criterio ha resultado ser prácticamente nula (exactamente un 0.3\%). Este resultado difiere de los encontrados por Özyurt (2015) y Tümkaya et al. (2009), que encontraron una asociación significativa entre disposición hacia el pensamiento crítico y destrezas de resolución de problemas. Estas diferencias podrían justificarse tanto por el tipo de participantes (estudiantes de secundaria vs. estudiantes universitarios), como por el tipo de prueba de resolución de problemas (problema de las pruebas PISA vs. cuestionario donde se evalúan destrezas genéricas de resolución de problemas -es decir, los estudiantes no han resuelto un problema, sino que han autoevaluado sus habilidades en resolución de problemas-).

\section{Conclusiones}

Se hace necesario subrayar, en primer lugar, las limitaciones de este trabajo. La principal limitación está localizada en la naturaleza y el tamaño de la muestra. La muestra seleccionada es pequeña y no es el resultado de un muestreo aleatorio. Además, en la variable nivel académico sólo han participado tres niveles de educación secundaria. Otras limitaciones pueden tener su origen en los instrumentos que se han empleado. Así pues, todas las conclusiones que se exponen seguidamente solo son válidas, en sentido estricto, para los estudiantes participantes y con los instrumentos empleados. 
Parece que, de todas las variables puestas en juego, el nivel académico o conjunción de la formación académica y del desarrollo evolutivo de los estudiantes, que supone una mejora de los conocimientos conceptuales y de los conocimientos y estrategias vinculadas con la resolución de problemas, así como el incremento de otras variables cognitivas (destrezas metacognitivas, capacidad de la memoria de trabajo, etc.) y motivacionales (autoeficacia, emociones positivas, etc.), es la que más peso tiene en la resolución de problemas académicos. El género no ha mostrado efecto alguno sobre el desempeño en la resolución de problemas, y la disposición hacia el pensamiento crítico tan solo se ha destacado por su coeficiente de correlación positivo con el desempeño en la resolución de problemas, bajo y no significativo. De este modo, ha dejado entrever que puede aportar alguna cosa a la resolución de problemas.

Así pues, y a pesar que en la literatura se incide en la interrelación entre resolución de problemas y pensamiento crítico, en esta ocasión no se ha evidenciado tal vínculo. Se necesitan más estudios sobre esta cuestión y también instrumentos sencillos y fiables que nos permitan acercarnos al pensamiento crítico de los estudiantes. Asimismo, el tipo de problemas que resuelven los estudiantes también es decisivo. Los problemas académicos usuales (problemas no reales, donde se proporcionan todos los datos necesarios, el procedimiento de resolución es conocido, y el objetivo está claramente definido) no dejan mucho margen, en sentido estricto, al pensamiento crítico. Se debe investigar si en problemas reales y más abiertos el pensamiento crítico tiene un papel más relevante.

\section{Referencias bibliográficas}

Alabau, J., Solaz-Portolés, J. J., \& Sanjosé, V. (2020). Relación entre creencias sobre resolución de problemas, creencias epistemológicas, nivel académico, sexo y desempeño en resolución de problemas: un estudio en educación secundaria. Revista Eureka sobre Enseñanza y Divulgación de las Ciencias, 17(1), 1102. DOI: https://doi.org/10.25267/Rev_Eureka_ensen_divulg_cienc.2020.v17.i1.1102

Butler, H. A. (2012). Halpern Critical Thinking Assessment predicts realworld outcomes of critical thinking. Applied Cognitive Psychology, 26(5), 721-729. DOI: https://doi.org/10.1002/acp.2851

Carvalho, C., Fíuza, E., Conboy, J., Fonseca, J., Santos, J. S., Gama, A. P., \& Salema, M. H. (2015). Critical thinking, real life problems and feedback in the sciences classroom. Journal of Turkish Science Education, 12(2), 21-31.

Docktor, J. y Heller, K. (2008). Gender differences in both force concept inventory and introductory physics performance. En C. Henderson, M. Sabella, y L. Hsu, Physics Education Research Conference 2008 (pp. 15-18). Melville, NY: American Institute of Physics.

Ennis, R. H. (1987). A taxonomy of critical thinking dispositions and habilities. In J. B. Baron \& R. J. Sternberg (Eds.), Teaching thinking skills theory and practice (pp. 9-26). New York: W. H. Freeman and Company.

Facione, P. A. (1990). The California critical thinking skills test-College level. Technical report \#2. Factors predictive of CT skills. Millbrae, CA: California Academic Press. Recuperado de https://files.eric.ed.gov/fulltext/ ED327550.pdf

Fisher, A. (2011). Critical thinking: an introduction (2nd Edition). Cambridge: Cambridge University Press.

Friede, C. R., Irani, T. A., Rhoades, E. B., Fuhrman, N. E., \& Gallo, M. (2008). It's in the Genes: Exploring Relationships between Critical Thinking and Problem Solving in Undergraduate Agriscience Students' Solutions to Problems in Mendelian Genetics. Journal of Agricultural Education, 49(4), 25-37.

Gallagher, A. M., De Lisi, R., Holst, P. C., McGillicuddy-De Lisi, A. V., Morely, M., y Cahalan,C. (2000). Gender differences in advanced mathematical problem solving. Journal of Experimental Child Psychology, 75(3), 165190. DOI: https://doi.org/10.1006/jecp.1999.2532

García-Gallego, P., Sanjosé, V., y Solaz-Portolés, J. J. (2015). Efectos de las características del problema, captación de su estructura y uso de analogías sobre el éxito de los estudiantes de secundaria en la resolución de problemas. Teoría de la Educación. Revista Interuniversitaria, 27(2), 221-244. DOI: http://dx.doi. org/10.14201/teri.13850 
Halpern, D. F. (2003). Thought and knowledge: An introduction to critical thinking (4th Edition). Mahwah, NJ: Erlbaum.

Hyde, J. S., Lindberg, S. M., Linn, M. C., Ellis, A. B., y Williams, C. C. (2008). Gender similarities characterize math performance. Science, 321(5888), 494-495. DOI: http://dx.doi.org/10.1126/science.1160364

Hyde, J. S., y Mertz, J. E. (2009). Gender, culture, and mathematics performance. Proceedings of the National Academy of Sciences, 106(22), 8801-8807. DOI: http://dx.doi.org/10.1073/pnas.0901265106

INECSE (2005). Pisa 2003. Pruebas de matemáticas y de solución de problemas. Madrid: MEC. Recuperado de:

http://educalab.es/inee/evaluaciones-internacionales/preguntasliberadas-pisa-piaac/enlaces

Kawashima, N., \& Shiomi, K. (2007). Factors of the thinking disposition of Japanese high school students. Social Behavior and Personality: An International Journal, 35(2), 187-194. DOI: https://doi.org/10.2224/ sbp.2007.35.2.187

Ku, K. Y., \& Ho, I. T. (2010). Metacognitive strategies that enhance critical thinking. Metacognition and Learning, 5(3), 251-267. DOI: https://doi.org/10.1007/s11409-010-9060-6

Kuhn, D. (1999). A developmental model of critical thinking. Educational Researcher, 28(2),16-46.

Lee, L. K. W., Tang, W. U., Goh, N. K., \& Chia, L. S. (2001). Cognitive variables in problem solving in stoichiometry. Chemistry Education Research and Practice, 2(3), 285-301. DOI: https://doi.org/10.1039/B1RP90029C

Lindberg, S. M., Hyde, J. S., Petersen, J. L., y Linn, M. C. (2010). New trends in gender and mathematics performance: a meta-analysis. Psychological Bulletin, 136(6), 1123. DOI: https://doi.org/10.1037/a0021276

Magno, C. (2010). The role of metacognitive skills in developing critical thinking. Metacognition and Learning, 5(2), 137-156. DOI: https://doi.org/10.1007/s11409-010-9054-4

Mugarra-Soldevila, I., Solaz-Portolés, J. J., y Caurín-Alonso, C. (2014). Efectos en estudiantes de secundaria de la utilización de símbolos que señalizan la dificultad de los problemas. Revista Electrónica Iberoamericana sobre Calidad, Eficacia y Cambio en Educación, 12(3), 93-108.

Norris, S. P. (1994). The meaning of critical thinking test performance: The effects of abilities and dispositions on scores. In D. Fasko (Ed.), Critical thinking: Current research, theory, and practice (pp. 315-329). Dordrecht, The Netherlands: Kluwer

Phillips, V., \& C. Bond (2004). Undergraduates' Experience with Critical Thinking. Higher Education Research \& Development, 23(3), 277-294. DOI: https://doi.org/10.1080/0729436042000235409

Özyurt, Ö. (2015). Examining the Critical Thinking Dispositions and the Problem Solving Skills of Computer Engineering Students. Eurasia Journal of Mathematics, Science \& Technology Education, 11(2), 353-361. DOI: https://doi.org/10.12973/eurasia.2015.1342a

Pellicer, I., Solaz-Portolés, J. J., \& Sanjosé, V. (2019). Emociones en ciencias y rendimiento en resolución de problemas: efectos del nivel académico y del género. En T. Sola, M. García, A. Fuentes, A. M. Rodríguez-García, \& J. López (Eds.), Innovación Educativa en la Sociedad Digital (pp. 2062-2074). Madrid: Editorial Dykinson.

Phan, H. P. (2010). Critical thinking as a self-regulatory process component in teaching and learning. Psicothema, 22(2), 284-292.

Pithers, R. T., \& Soden, R. (2000). Critical thinking in education: A review. Educational Research, 42(3), 237-249. DOI: https://doi.org/10.1080/001318800440579

Pushkin, D. (2007). Critical thinking and problem solving-the theory behind flexible thinking and skills development. Journal of Science Education, 8(1), 13-17.

Ricketts, J. C., \& Rudd, R. D. (2005). Critical thinking skills of selected youth leaders: The efficacy of critical thinking dispositions, leadership, and academic performance. Journal of Agricultural Education, 46(1), 32-43. 
Schraw, G., Crippen, K. J., \& Hartley, K. (2006). Promoting self-regulation in science education: Metacognition as part of a broader perspective on learning. Research in Science Education, 36(1-2), 111-139. DOI: https://doi. org/10.1007/s11165-005-3917-8

Solaz-Portolés, J. J., \& Sanjosé, V. (2008). Conocimientos y procesos cognitivos en la resolución de problemas de ciencias: consecuencias para la enseñanza. Magis, Revista internacional de Investigación en Educación, 1(1), 147-162.

Tsai, K. C. (2019). Investigating the Empirical Links between Creative and Critical Thinking. Psychology, Society, \& Education, 11(3), 267-280. DOI: https://doi.org/10.25115/psye.v10i1.1064

Ten Dam, G., \& Volman, M. (2004). Critical thinking as a citizenship competence: teaching strategies. Learning and Instruction, 14(4), 359-379. DOI: https://doi.org/10.1016/j.learninstruc.2004.01.005

Tümkaya, S., Aybek, B., \& Aldaş, H. (2009). An Investigation of University Students' Critical Thinking Disposition and Perceived Problem Solving Skills. Eurasian Journal of Educational Research (EJER), 36, 57-74.

Valenzuela, J., Nieto, A., \& Saiz, C. (2011). Critical thinking motivational scale: A contribution to the study of relationship between critical thinking and motivation. Electronic Journal of Research in Educational Psychology, 9(2), 823-848. Recuperado de http://repositorio.ual.es/bitstream/handle/10835/819/Art_24_588. pdf?sequence $=1$

Van der Stel, M., Veenman, M. V., Deelen, K., \& Haenen, J. (2010). The increasing role of metacognitive skills in math: A cross-sectional study from a developmental perspective. ZDM, 42(2), 219-229. DOI: https://doi. org/10.1007/s11858-009-0224-2

Veenman, M. V., \& Spaans, M. A. (2005). Relation between intellectual and metacognitive skills: Age and task differences. Learning and Individual Differences, 15(2), 159-176. DOI: https://doi.org/10.1016/ j.lindif.2004.12.001

Zhu, Z. (2007). Gender differences in mathematical problem solving patterns: A review of literature. International Education Journal, 8(2), 187-203.

Zoller, U., Ben-Chaim, D., Ron, S., Pentimalli, R., Scolastica, S., Chiara, M. S., \& Borsese, A. (2000). The disposition toward critical thinking of high school and university science students: an interintra Israeli-Italian Study. International Journal of Science Education, 22(6), 571-582. DOI: https://doi. org/10.1080/095006900289679 